\title{
Histone deacetylase inhibitor scriptaid induces cell cycle arrest and epigenetic change in colon cancer cells
}

\author{
EUN JU LEE ${ }^{1}$, BO BIN LEE ${ }^{1}$, SOON-JA KIM ${ }^{1}$, YONG-DOO PARK ${ }^{2}$, \\ JOOBAE PARK ${ }^{1,3}$ and DUK-HWAN KIM ${ }^{1,3}$ \\ ${ }^{1}$ Department of Molecular Cell Biology, Sungkyunkwan University School of Medicine, \\ Suwon 440-746, Korea; ${ }^{2}$ Institute of Biotechnology and Medicine, Tsinghua University, \\ Zhejang 314100, P.R. China; ${ }^{3}$ Center for Genome Research, Samsung Biomedical \\ Research Institute, Sungkyunkwan University School of Medicine, Seoul 135-710, Korea
}

Received May 14, 2008; Accepted July 23, 2008

DOI: 10.3892/ijo_00000063

\begin{abstract}
Histone deacetylase inhibitors (HDACIs) are involved in cell growth, apoptosis and differentiation. This study aimed to investigate the effects of HDACI scriptaid on histone modification, demethylation, cell growth, cell cycle and apoptosis in the RKO colorectal cancer cell line and screening for scriptaid-induced genes. RKO cells were treated with 5-aza-2'-deoxycytidine (5-aza-dC), trichostatin A (TSA) or scriptaid at different concentrations. Histone modification and methylation status of a silenced p16 gene were analyzed using chromatin immunoprecipitation and methylation-specific PCR, respectively. Flow cytometry was performed for the analysis of cell cycle and apoptosis. Scriptaid-induced expression was analyzed using Human OneArray ${ }^{\mathrm{TM}}$ chip. Scriptaid resulted in the demethylation and re-expression of a hypermethylated $p 16$ gene along with 5 -aza-dC synergistically in the RKO cells, but not alone. Scriptaid induced modifications of core histone tails important in euchromatin structure: increases in acetyl-H3-K9 and dimethyl-H3-K4 and a decrease in dimethyl-H3-K9. Cell growth was inhibited by scriptaid in a dose-dependent manner. Cell cycle analysis showed that scriptaid induced G1 arrest at 0.5 and $1.0 \mu \mathrm{M}$ concentrations and G1 and G2/M arrest at 2.0 $\mu \mathrm{M}$. Scriptaid did not have a significant effect on apoptosis in RKO cells. An
\end{abstract}

Correspondence to: Dr Duk-Hwan Kim, Center for Genome Research, Samsung Biomedical Research Institute, Rm B155, \#50 Ilwon-dong, Kangnam-Ku, Seoul 135-710, Korea

E-mail: dukhwan.kim@samsung.com

Abbreviations: 5-aza-dC, 5-aza-2'-deoxycytidine; TSA, trichostatin A; HDACI, histone deacetylase inhibitor; MSP, methylation-specific polymerase chain reaction; EMEM, Eagle's minimum essential medium; ChIP, chromatin immunoprecipitation

Key words: scriptaid, growth suppression, histone, methylation, colorectal cancer altered expression of 278 genes was observed in RKO cells in response to scriptaid treatment. In conclusion, the present study suggests that scriptaid may be effective in growth suppression and cell cycle arrest and in the reversal of repressive chromatin marks at the promoter region of a hypermethylated $p 16$ gene in colorectal cancer.

\section{Introduction}

Aberrant methylation of $\mathrm{CpG}$ island at a promoter region of tumor suppressor genes is an epigenetic change that induces transcriptional silencing of genes. The aberrant methylation in a number of tumor suppressor genes has been reported in a variety of tumors and is now widely recognized as a contributing factor in human tumorigenesis (1). Transcriptional repression by $\mathrm{CpG}$ island hypermethylation is closely linked to modifications of histone tails (2-4). Deacetylation of the histone H3-K9 lysine residues by histone deacetylases (HDACs) results in a positively charged histone tail by its effect at the amino-terminal lysine residues of core histones and subsequently leads to the remodeling of nucleosomes, condensation of chromatin structure and $\mathrm{CpG}$ island hypermethylation (5-7). Accordingly, inhibition of HDAC activity plays a role in the accumulation of acetylated core histones, leading to a more relaxed chromatin conformation and the transcriptional activation of a limited number of target genes suppressed by $\mathrm{CpG}$ island hypermethylation.

HDAC inhibitors (HDACIs) are a novel and promising class of chemotherapeutic agents that can inhibit the activity of HDACs and suppress the growth of tumor cells. In addition, HDACIs induce extrinsic and/or intrinsic apoptosis and differentiation and possess antiangiogenic and antilymphangiogenic properties (reviewed in ref. 8). A diverse group of HDACIs have been discovered and their effects on cancer cells are known to differ with regards to their antitumor activity, toxicity and stability (9). These HDACIs include cyclic and non-cyclic hydroxamic acids (i.e. trichostatin A, oxamflatin, proxamide, SAHA and suberoyl-3-aminopyridineamide hydroxamic acid), short-chain fatty acids (i.e. valproic acid, sodium butyrate and phenylbutyrate), cyclic 
peptides or tetrapeptides (i.e. Trapoxin, apicidin and FK-228), ketones, cyclic tetrapeptide and benzamides (i.e. MS-275). Some HDACIs are in clinical trials in cancer patients.

Scriptaid, the 6-[1,3-dioxo- $1 H, 3 H$-benzo(de)isoquinolin2-yl]-hexanoic acid hydroxyamide, was identified by screening a library of 16,320 compounds (DIVERset, Chembridge, San Diego, CA) using a high-throughput system based on a stably integrated transcriptional reporter. The drug is considered to be a novel HDACI with robust activity and relatively low toxicity compared to TSA (10). Scriptaid has been reported to inhibit the growth and induce differentiation and/or apoptosis in a variety of cancer cells such as breast, endometrial and ovarian cancers $(11,12)$, but little is known of the overall effect of this drug in colorectal cancer. This study was aimed at investigating the effect of scriptaid on the epigenetic change and re-expression of a hypermethylated p16 gene and on cell growth in a type of colorectal cancer cell. In addition, genes induced by scriptaid were also screened in the cells.

\section{Materials and methods}

Cell culture. The RKO colorectal cancer cell line was obtained from the American Type Culture Collection (Manassas, VA). The cells $\left(2.0 \times 10^{5}\right)$ in most experiments were seeded in six-well plates and allowed to attach for at least $16 \mathrm{~h}$ before the addition of drugs such as 5-aza-2'-deoxycytidine (5-aza$\mathrm{dC}$ ), trichostatin A (TSA) or scriptaid. RKO cells were grown in Eagle's Minimum Essential Medium (EMEM, Cambrex, Walkersville, MD) media supplemented with $10 \%$ fetal bovine serum (Hyclone, Logan, UT), 1\% antibiotic-antimycotic (Gibco, New York, NY), $1.0 \mathrm{mM}$ of sodium pyruvate (Sigma-Aldrich, St. Louis, MO) and 1\% MEM non-essential amino acid solution (Sigma-Aldrich) at $37^{\circ} \mathrm{C}$ in an atmosphere of $5 \% \mathrm{CO}_{2}$.

In vitro growth assay. Cells $\left(2.0 \times 10^{5}\right)$ were seeded in six-well plates in triplicate and treated with $0.1 \mu \mathrm{M} 5$-aza-dC or $1.0 \mu \mathrm{M}$ scriptaid alone, or $0.1 \mu \mathrm{M} 5$-aza-dC along with either $0.3 \mu \mathrm{M}$ TSA or $1.0 \mu \mathrm{M}$ scriptaid. Cells were harvested with trypsin after 24,48 and $72 \mathrm{~h}$ of exposure to each drug. Cells were counted using a hemacytometer and cell viability was assessed by trypan blue exclusion. For the analysis of a dose-dependent effect of scriptaid on cell growth, RKO cells were incubated at different concentrations of scriptaid $(0,0.5,1.0$ and $2.0 \mu \mathrm{M})$ for 24,48 and $72 \mathrm{~h}$.

Methylation-specific polymerase chain reaction (MSP). Cells were cultured with either $0.1 \mu \mathrm{M} 5$-aza-dC or $1.0 \mu \mathrm{M}$ scriptaid as indicated for 12, 24 or $48 \mathrm{~h}$. Cells were harvested at the indicated times and washed in ice-cold phosphate-buffered saline (PBS). Genomic DNA was obtained from the cell lines using DNeasy Blood \& Tissue kit (Qiagen, Valencia, CA) according to the manufacturer's protocol. For the analysis of the combined effect of 5-aza-dC and scriptaid (or TSA) on demethylation of the hypermethylated p16 gene, cells were also cultured with 5-aza-dC for $24 \mathrm{~h}$ and co-incubated with scriptaid (or TSA) for an additional $24 \mathrm{~h}$. The methylation status of $\mathrm{CpG}$ island at the promoter region of the $p 16$ gene was determined by methylation-specific PCR (MSP), as previously described using two pairs of primers (13): one for the unmethylated promoter and another for the methylated promoter. The primer sequences used for MSP are shown in Table I. CpGenome ${ }^{\mathrm{TM}}$ Universal Methylated DNA (Chemicon, Temecula, CA) was subject to bisulfite modification and used as a positive-control for the methylated alleles. Bisulfitemodified normal DNA served as a positive control for the unmethylated alleles and negative control samples without DNA were included in every PCR experiment.

Reverse transcription PCR (RT-PCR). Cells were cultured with either $0.1 \mu \mathrm{M} 5$-aza-dC or $1.0 \mu \mathrm{M}$ scriptaid for $48 \mathrm{~h}$, or with $0.1 \mu \mathrm{M} 5$-aza-dC for $24 \mathrm{~h}$, which was followed by co-incubation with $1.0 \mu \mathrm{M}$ scriptaid (or $0.3 \mu \mathrm{M}$ TSA) for an additional $24 \mathrm{~h}$. After $48 \mathrm{~h}$ of culture, cells were harvested and washed in ice-cold PBS. For analyzing dose-dependence of scriptaid on re-expression of the hypermethylated $p 16$, RKO cells were also cultured with different concentrations of scriptaid $(0.1,0.5$ and $1.0 \mu \mathrm{M})$. Total RNA was isolated from RKO cells using RNeasy ${ }^{\circledR}$ Mini kit (Qiagen, Valencia, CA) according to the manufacturer's instructions. RNA was resuspended in diethyl pyrocarbonate-treated water and was quantitated by spectrophotometer. RT-PCR was carried out in a tube containing $0.5 \mu \mathrm{g}$ of total RNA and p16-specific primers (Table I) at a final concentration of $0.6 \mu \mathrm{M}$ using a one step RT-PCR kit (Qiagen) according to the manufacturer's protocols. PCR products were quantitated with the GeneAmp PCR System 2700 (Applied Biosystems, Foster City, CA).

Chromatin immunoprecipitation. Cells were cultured with either $0.1 \mu \mathrm{M} 5$-Aza-dC or $1.0 \mu \mathrm{M}$ scriptaid for $48 \mathrm{~h}$, or with $0.1 \mu \mathrm{M} 5$-aza-dC for $24 \mathrm{~h}$, which was followed by coincubation with $1.0 \mu \mathrm{M}$ scriptaid (or $0.3 \mu \mathrm{M}$ TSA) for an additional $24 \mathrm{~h}$. After $48 \mathrm{~h}$ of culture, chromatin immunoprecipitation (ChIP) was performed using a ChIP assay kit (Upstate Biotechnology, Lake Placid, NY) according to the manufacturer's recommendations. Antibodies used for ChIP were anti-acetyl-histone H3 (Lys9), anti-dimethyl-histone H3 (Lys4) and anti-dimethyl-histone H3 (Lys9) (all from Upstate Biotechnology). Primer sequences for p16 amplification are listed in Table I.

Flow cytometry analysis of cell cycle and apoptosis. RKO cells $\left(2.0 \times 10^{5}\right)$ were seeded in six-well plates in triplicate and allowed to attach for at least $16 \mathrm{~h}$ before the addition of $1.0 \mu \mathrm{M}$ scriptaid. The cells were harvested and washed in ice-cold PBS at $48 \mathrm{~h}$ of exposure. For cell cycle analysis, the cells were then fixed with $70 \%$ ice-cold ethanol for $24 \mathrm{~h}$ and DNA was stained with propidium iodide $(50 \mu \mathrm{g} / \mathrm{ml})$ containing $5 \mathrm{mg} / \mathrm{ml}$ RNase (Boehringer Ingelheim, Ingelhein, Germany) at a dilution of 1:100 in darkness. Apoptosis was analyzed using an Annexin-V-FITC Apoptosis Detection kit II (Calbiochem, San Diego, CA) after washing the cells with ice-cold PBS. Fluorescence for cell cycle and apoptosis distribution was detected by a FACScan flow cytometer (Becton-Dickson, San Jose, CA) and obtained data was analyzed using the ModFit LT version 3.0 (Verity Software House, Topsham, NE). At least 10,000 cells were examined for each sample and the test was run in duplicate for each subject. 
Table I. Primer and probe sequences.

Sense $\left(5^{\prime} \rightarrow 3^{\prime}\right)$

Antisense $\left(5^{\prime} \rightarrow 3^{\prime}\right)$

\begin{tabular}{lll}
\hline & \multicolumn{1}{c}{ Sense $\left(5^{\prime} \rightarrow 3^{\prime}\right)$} & Antisense $\left(5^{\prime} \rightarrow 3^{\prime}\right)$ \\
\hline MSP $(p 16)$ & TTATTAGAGGGTGGGGCGGATCGC & \\
$\mathrm{M}^{\mathrm{a}}$ & TTATTAGAGGGTGGGGTGGATTGT & CACCTCGACCGACCG \\
$\mathrm{U}^{\mathrm{a}}$ & & CCACCTAAATCAACCTCCAACCA \\
RT-PCR & CAACGCACCGAATAGTTACGG & \\
p16 & CAGCCGAGCCACATCGCTCAGACA & CGCCAGTTGGGCTCCG \\
GAPDH & & \\
ChIP & & TGAGCTGTTGTCATACTTCTC \\
$p 16$ & AGACAGCCGTTTTACACGCAG & CACCGAGAAATCGAAATCACC \\
\hline
\end{tabular}

${ }^{\mathrm{a}} \mathrm{M}$ and $\mathrm{U}$ indicate primers for methylated and unmethylated alleles of the $p 16$ gene, respectively.

Gene expression analysis. RKO Cells were incubated with $1.0 \mu \mathrm{M}$ scriptaid for $48 \mathrm{~h}$. Total RNA was extracted using the RNeasy Mini kit (Qiagen) according to the manufacturer's instructions. RNA quantity and purity were assessed by measurement of $\mathrm{OD}_{260 / 280}$ using a NanoDrop ${ }^{\circledR}$ spectrophotometer. The quality was verified by the integrity of $28 \mathrm{~S}$ and 18S rRNA using the Agilent Total RNA Nano chip assay on a Model 2100 Bioanalyzer (Agilent Technologies, Santa Clara, CA). Ideally, the intensity of $28 \mathrm{~S}$ band should be twice the intensity of $18 \mathrm{~S}$ band. Expression was analyzed using Human OneArray $^{\mathrm{TM}}$ (Phalanx Biotech Group, Palo Alto, CA) according to the manufacturer's recommendations. Briefly, fluorescence-labeled cDNA for oligo microarray analysis was prepared by amplification of $15 \mu \mathrm{g}$ of high-quality total RNA in the presence of aminoallyl-UTP followed by the coupling of Cy3 or Cy5 dye using CyScribe ${ }^{\mathrm{TM}}$ First-Strand cDNA Labeling kit (Amersham Pharmacia, Uppsala, Sweden). The labeled chip was hybridized with the fluorescently labeled cRNA at $42^{\circ} \mathrm{C}$ for $16 \mathrm{~h}$ and then washed. DNA chips were scanned using GenePix 4000B (Axon Instruments, Unio City, CA). Scanned images were analyzed with GenePix Pro 3.0 software (Axon Instruments, Union City, CA) to obtain gene expression ratios. Transformed data were normalized using the Lowess procedure (14). The normalized data were used for clustering analysis. Clustering analysis was performed using the Cluster and TreeView programs (http://rana.lbl.gov) to provide a graphical display of the expression patterns.

\section{Results}

Scriptaid alone did not induce demethylation and re-expression of silenced p16. The effect of scriptaid on a hypermethylated p16 gene was first evaluated by treating RKO cells with $0.1 \mu \mathrm{M} 5$-aza-dC or $1.0 \mu \mathrm{M}$ scriptaid for $48 \mathrm{~h}$. The 5 -aza-dC resulted in demethylation of the hypermethylated p16 at $12 \mathrm{~h}$ after treatment and the degree of demethylation increased with time. However, scriptaid alone did not result in demethylation of the $p 16$ gene even after $48 \mathrm{~h}$ (Fig. 1A). For the analysis of combined effect of 5-aza-dC and scriptaid (or TSA), cells were cultured with $0.1 \mu \mathrm{M} 5$-aza-dC for $24 \mathrm{~h}$ and co-incubated with $1.0 \mu \mathrm{M}$ scriptaid (or $0.3 \mu \mathrm{M}$ TSA) for an additional $24 \mathrm{~h}$. The addition of scriptaid in medium treated with 5-aza-dC caused demethylation of the pl6 gene synergistically (Fig. 1B). The demethylating effect of scriptaid in the presence of 5aza-dC was similar to that evident following co-treatment with TSA and 5-aza-dC. The re-expression of the silenced p16 was evaluated after treatment of cells with $0.1 \mu \mathrm{M} 5$-aza$\mathrm{dC}$ or $1.0 \mu \mathrm{M}$ scriptaid for $48 \mathrm{~h}$ (Fig. 1C). Furthermore, the cells were also incubated for another $24 \mathrm{~h}$ in the presence of $0.3 \mu \mathrm{M}$ TSA or $1.0 \mu \mathrm{M}$ scriptaid following $24 \mathrm{~h}$ of initial 5 -aza-dC treatment. Scriptaid induced reactivation of the silenced p16 along with 5-aza-dC, but not alone. In addition, the scriptaid $(0.1,0.5$ and $1.0 \mu \mathrm{M})$ at different concentrations resulted in the reactivation of hypermethylated $p 16$ gene in a dose-dependent manner along with $0.1 \mu \mathrm{M}$ 5-aza-dC (Fig. 1D). The synergistic effect of scriptaid on reactivation of silenced p16 in the presence of 5-aza-dC was similar to that of TSA at different concentrations.

Scriptaid induces histone modifications. It is known that there is a relationship between key elements of the histone code and DNA methylation. The effect of scriptaid on a change of the histone code components at the $p 16$ promoter was analyzed using chromatin immunoprecipitation (ChIP) in RKO cells (Fig. 2A) and quantitative levels (Fig. 2B) of modified histones in RKO cells treated with $\operatorname{drug}(\mathrm{s})$ were measured by dividing [band intensity of ChIP PCR in RKO cells treated with $\operatorname{drug}(\mathrm{s}) \div$ band intensity of input in the cells] by (band intensity of ChIP PCR in control $\div$ band intensity of input in control). The quantitative analyses revealed that the levels of acetyl-H3-K9 and dimethyl-H3-K4 at the p16 promoter after $1.0 \mu \mathrm{M}$ scriptaid treatment for $48 \mathrm{~h}$ increased 1.7 and 1.5 times compared to the control, respectively. The application of $0.1 \mu \mathrm{M}$ 5-aza-dC alone also increased the levels 1.36 and 1.70 times, respectively. Scriptaid addition in the cells treated with 5-aza- dC synergistically increased the levels of acetylH3-K9 and dimethyl-H3-K4 at the p16 promoter (2.4- and 2.6-fold, respectively). In addition, scriptaid decreased dimethyl-H3-K9 levels 0.78 -fold compared to the control. However, the level of dimethyl-H3-K9 was not significantly decreased in response to 5 -aza-dC. These data suggest that scriptaid alone was able to evoke obvious changes in key parameters of the histone code at the promoter of the p16 gene in RKO cells. 
A.

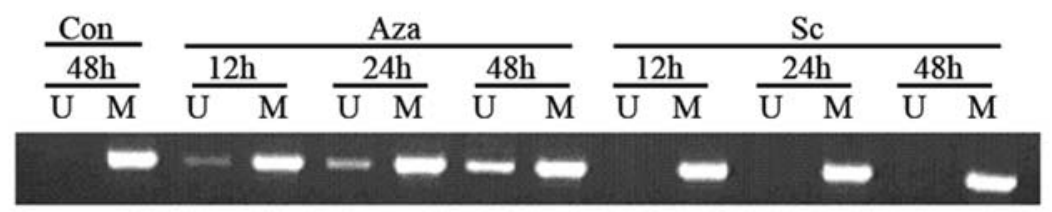

B.

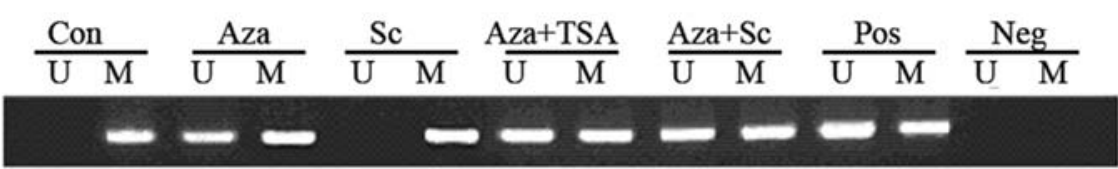

C.

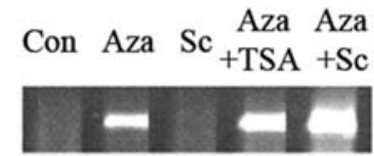

pl6

GAPDH

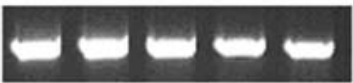

D.

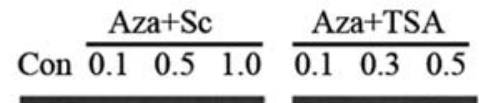

p16
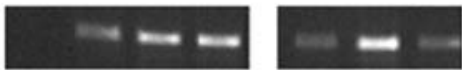

GAPDH

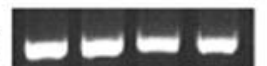

$\infty \pi$

Figure 1. Effects of scriptaid on demethylation, re-expression and histone modification of silenced p16 in RKO cells. (A) RKO cells were treated with $0.1 \mu \mathrm{M}$ 5 -aza-dC or $1.0 \mu \mathrm{M}$ scriptaid for the indicated times. Demethylation of a hypermethylated p16 gene was examined by methylation-specific PCR (MSP). (B) RKO cells were treated with $1.0 \mu \mathrm{M}$ scriptaid, $0.1 \mu \mathrm{M}$ 5-aza-dC alone or in combination with $1.0 \mu \mathrm{M}$ scriptaid or $0.3 \mu \mathrm{M}$ TSA for $48 \mathrm{~h}$. Methylation status of the $p 16$ gene was assessed by the MSP assay. 'Pos' represents the positive controls for the methylated (M) and unmethylated (U) allele. Negative control samples without DNA were included for each PCR. (C) Re-expression of silenced p16 was examined by RT-PCR after treating the cells with the indicated compounds for $48 \mathrm{~h}$. (D) Dose-dependence of scriptaid on re-expression of silenced p16 was assessed by RT-PCR after incubating cells for $48 \mathrm{~h}$ in combination with $0.1 \mu \mathrm{M}$ 5-aza-dC and scriptaid (or TSA) at different concentrations. Con, control; Aza, 5-aza-dC and Sc, scriptaid.

A.

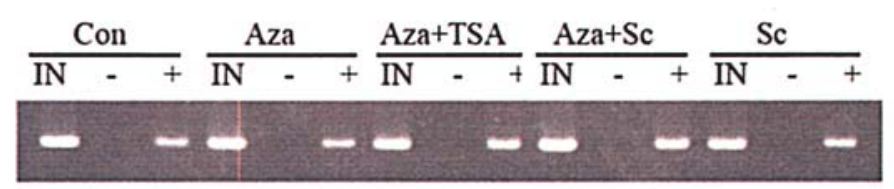

Acetyl-H3-K9

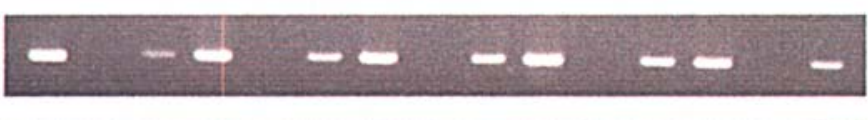

Dimethyl-H3-K4

Dimethyl-H3-K9

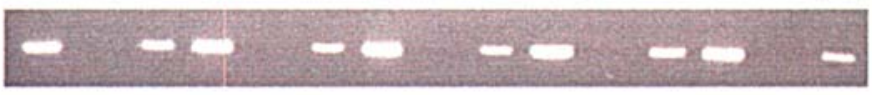

B.

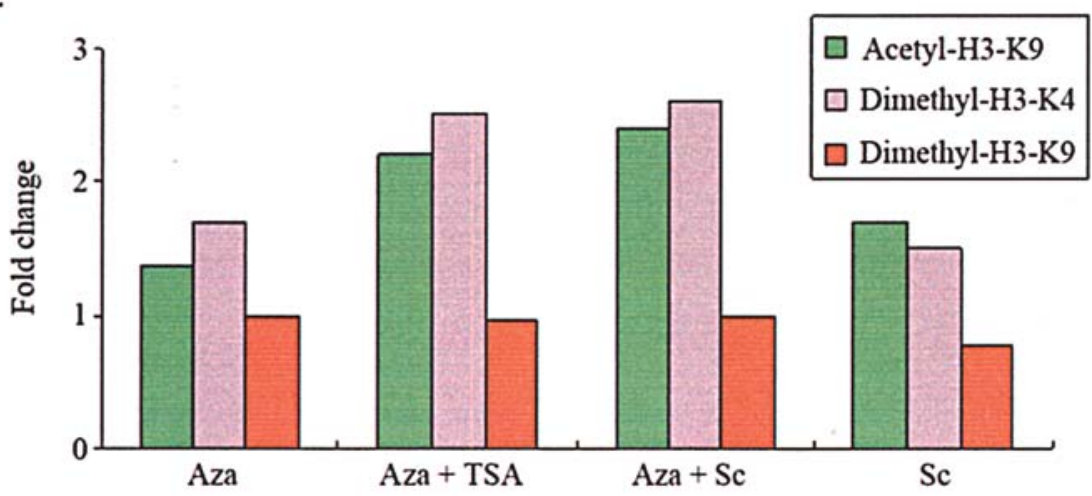

Figure 2. Effects of scriptaid on acetylation and demethylation of histones at the promoter of the p16 gene. (A) Histone modifications at the p16 promoter were measured by chromatin immunoprecipitation (ChIP) assay after treating cells with indicated drugs for $48 \mathrm{~h}$. Input DNA, before immunoprecipitation, was also subjected to PCR as a control. (B) Each ChIP experiment was repeated three times to confirm reproducibility of results and data shown are an average of relative amounts of accumulated histones at the p16 promoter compared to the control. IN, input; Con, control; Aza, 5-aza-dC and Sc, scriptaid.

Scriptaid inhibits cell growth. The effect of scriptaid on the growth of RKO cells in vitro was determined. The proportion of actively replicating cells in response to scriptaid significantly decreased in a time-dependent and a dose-dependent manner. The percentage of viable cells in the culture decreased $\sim 60 \%$ of control after $48 \mathrm{~h}$ of $1.0 \mu \mathrm{M}$ scriptaid treatment (Fig. 3A). 
A.

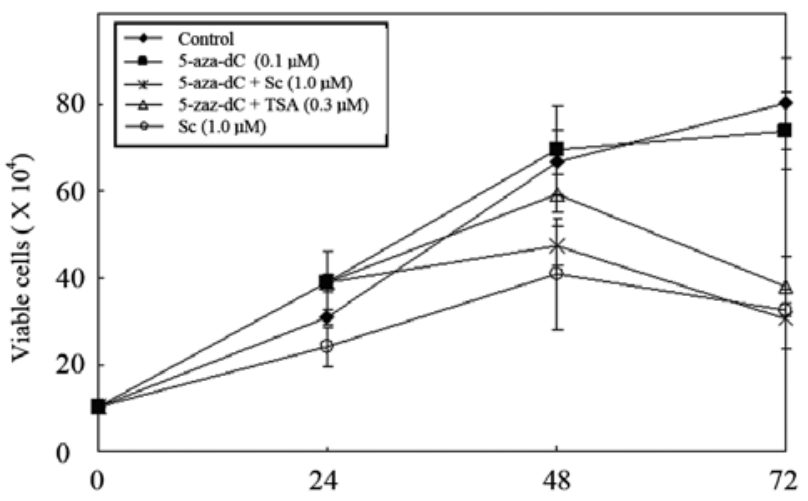

B.

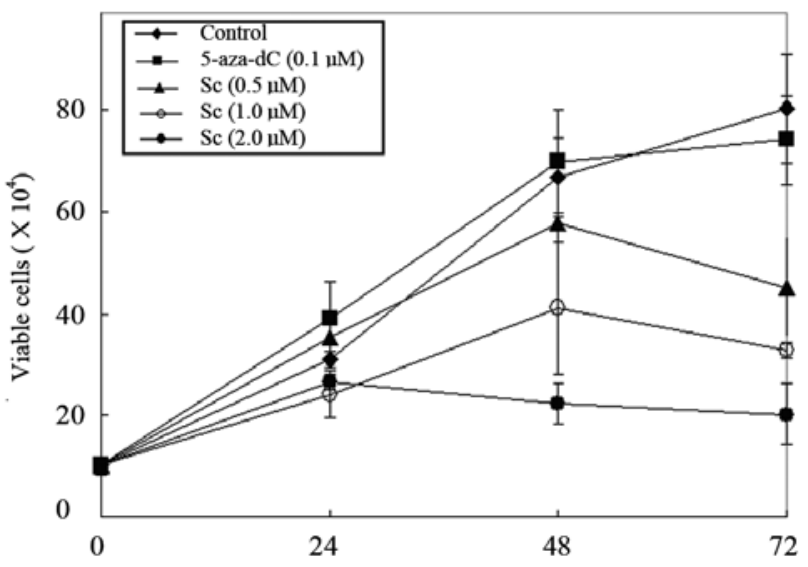

Figure 3. Induction of growth inhibition in RKO cells. (A) RKO cells were treated with $0.1 \mu \mathrm{M} 5$-aza-dC alone or in combination with $1.0 \mu \mathrm{M}$ scriptaid or $0.3 \mu \mathrm{M}$ TSA for different periods of time as indicated in the figure. (B) The effect of scriptaid on the viable cell number of RKO cells was assessed with increasing doses of scriptaid over time. At the end of the indicated time points, cell viability was assessed by trypan blue exclusion and viable cells were counted using a hemacytometer. The data shown are the mean \pm SD of three independent experiments.

However, $0.1 \mu \mathrm{M}$ 5-aza-dC did not have a growth suppressive effect. Co-treatment of $0.1 \mu \mathrm{M} 5$-aza-dC and $1.0 \mu \mathrm{M}$ scriptaid did not show a synergistic suppressive effect on cell growth compared to scriptaid alone. To test a dose-dependent effect of scriptaid on cell growth, RKO cells were treated for $72 \mathrm{~h}$ at different concentrations of scriptaid $(0.5,1.0$ and $2.0 \mu \mathrm{M})$. The growth suppressive effect of scriptaid was evident compared to the controls after $48 \mathrm{~h}$ of treatment and the degree of growth suppression was proportional to the concentration of scriptaid (Fig. 3B).

Scriptaid results in cell cycle arrest. Fluorescence-activated cell sorting (FACS) was carried out to analyze cell cycle profiling of scriptaid-treated cells (Fig. 4). The proportion of $\mathrm{S}$ phase cells at $48 \mathrm{~h}$ after scriptaid treatment was $30.4 \%$ at control and 16.9, 11.1 and $5.7 \%$ at scriptaid concentrations of $0.5,1.0$ and $2.0 \mu \mathrm{M}$, respectively. The reduction in $\mathrm{S}$ phase cells resulted in a concomitant change in $\mathrm{G} 1$ and G2/M phase cells. The proportion of cells containing $2 \mathrm{~N}$ amount of DNA, which represents $\mathrm{G} 1$ phase cells, was $63.9 \%$ at control and increased significantly up to $83.9 \%$ in the presence of $1.0 \mu \mathrm{M}$ scriptaid and $76.5 \%$ for $2.0 \mu \mathrm{M}$ scriptaid. Similarly, the fraction of cells with $4 \mathrm{~N}$ amount of DNA, which represents G2/M phase cells, increased significantly especially at high concentrations of $2.0 \mu \mathrm{M}$ scriptaid compared to control (17.8 vs. $5.6 \%$, respectively). These results suggest that the treatment of RKO cells with scriptaid resulted in the inhibition of cellular DNA synthesis leading to the arrest of cells in G1 at low concentrations and in the G1 and G2/M phase of the cell cycle at high concentrations.

Scriptaid does not induce apoptosis. The apoptotic alterations in scriptaid-treated RKO cells were assessed using Annexin-V staining for phosphatidylserine (PS) externalization. Fig. 5 shows the typical dotplots of Annexin-V and PI staining in which three distinctive cell populations were clearly seen. Early apoptosis fraction in the RKO cells at $48 \mathrm{~h}$ after culture increased slightly with concentrations of scriptaid compared to the control. At 1.0 and $2.0 \mu \mathrm{M}$ scriptaid, the fractions of early apoptosis (Annexin-V-positive, PInegative) were 1.01 and $1.00 \%$, respectively, whereas cells cultured without scriptaid had early apoptosis of $0.12 \%$. Scriptaid-treated cells also showed a decrease of late apoptosis fraction (Annexin-V-positive, PI-positive) compared with control samples at $48 \mathrm{~h}$. The control sample of RKO cells showed a late apoptosis fraction of $0.23 \%$ as compared with cells cultured with 1.0 and $2.0 \mu \mathrm{M}$ scriptaid, which showed late apoptosis fraction of 0.15 and $0.22 \%$, respectively. However, differences in early- and lateapoptosis between control and scriptaid-treated cells were not statistically significant $(\mathrm{P}=0.37$ and $\mathrm{P}=0.75$, respectively; Wilcoxon-rank sum test). These experiments suggest that RKO cells may not undergo apoptosis upon scriptaid treatment.

Identification of genes induced by scriptaid. Expression profiling using cDNA microarrays was carried out to identify genes induced by scriptaid in RKO cells. To assess the reliability of our microarray technique, we compared the expression levels of two genes (TIMP3 and hMLH1) methylated in RKO cells. The mRNA levels of the genes after scriptaid treatment were not detected in the microarray as well as RT-PCR (data not shown), indicating a high reliability for our microarray data. Of the total 30,968 genes analyzed, 122 genes were up-regulated and 156 genes were down-regulated with a mean expression value of 2.0 -fold or higher after $1.0 \mu \mathrm{M}$ scriptaid treatment for $48 \mathrm{~h}$. The majority of the genes with an altered expression of a 2.0 -fold or higher were determined to be involved mainly in cell cycle, tumor metastasis, metabolism, inflammation, drug resistance, apoptosis and signaling pathways. The genes involved in cell cycle and metastasis among the genes regulated by scriptaid are listed in Table II.

Scriptaid differentially regulated a number of cell cycle-regulating genes in RKO cells. Scriptaid usually down-regulated genes involved in cell cycle progression and up-regulated genes associated with cell cycle inhibition. Twelve genes were up-regulated and 19 genes were downregulated in response to scriptaid. The expression levels of G0/G1 switch 2 (GOS2) and cyclin-dependent kinase inhibitor 2D (CDKN2D) increased 3.1- and 3.0-fold, respectively, in cells treated with scriptaid. Centrin EF-hand protein 2 
A.

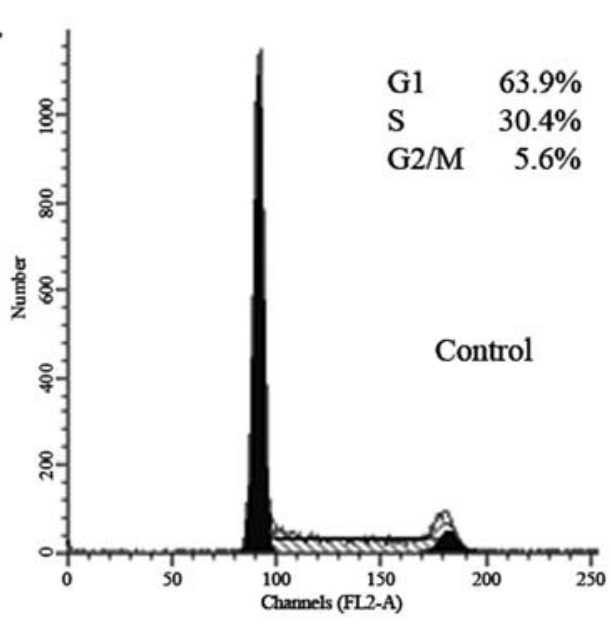

C.

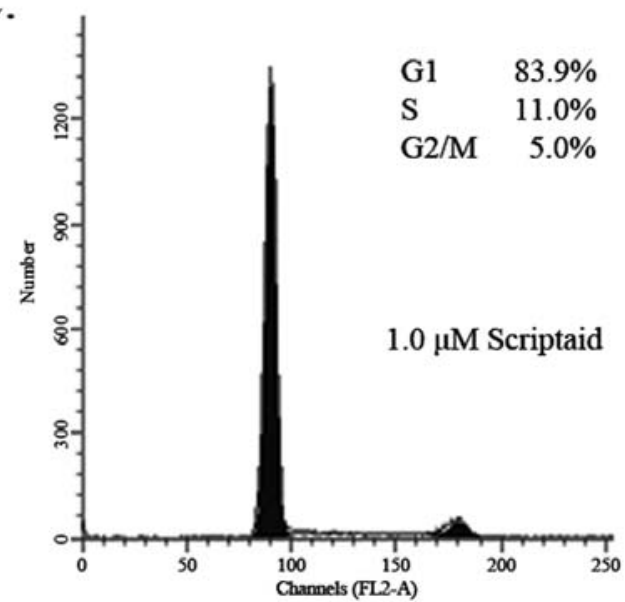

B.

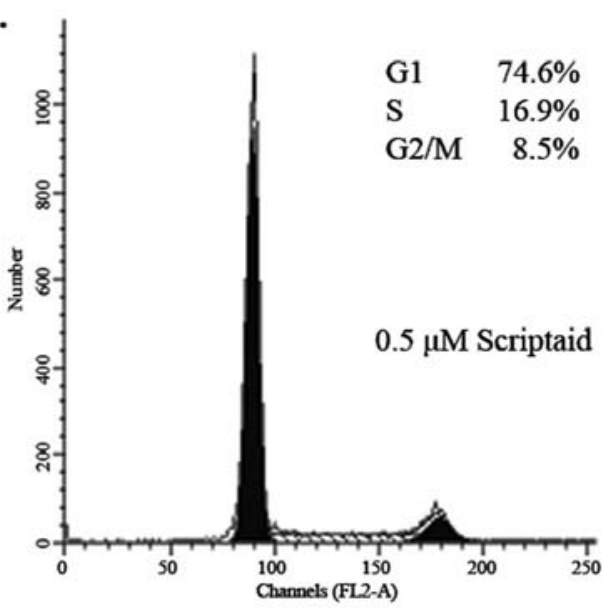

D.

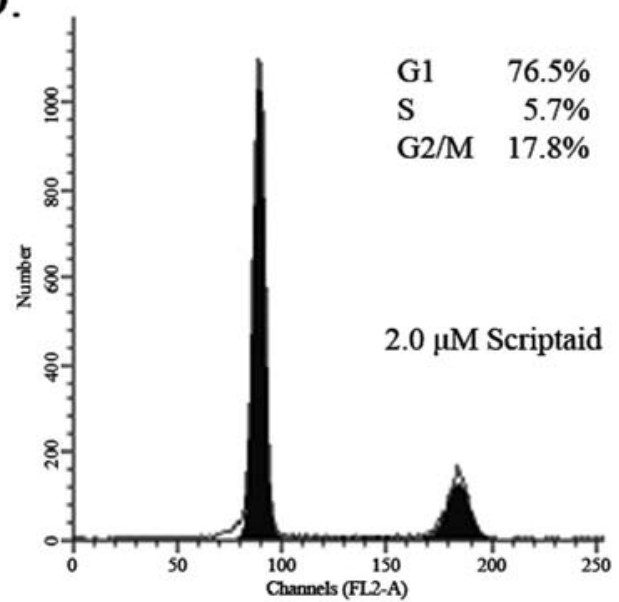

Figure 4. FACS analysis of RKO cells. Cells were cultured with scriptaid doses of 0, 0.5, 1.0 and $2.0 \mu \mathrm{M}$. Cells were harvested after 48 h of exposure to the scriptaid and stained with propidium iodine and the cell cycle was analyzed by flow cytometry. Results are shown as the percent of cells in G1, G2/M and S-phases of the cell cycle.

(CENT2), growth-arrest-specific 1 (GAS1), dual specificity phosphatase 6 (DUSP6) and septin 3 (SEPTIN3) were increased by 2 -fold or higher in response to scriptaid. Similarly, scriptaid down-regulated cell cycle progression genes including cyclin B2, cyclin E2, CDK3 and E2F transcription factor 3 .

Scriptaid up-regulated a set of metastasis suppressor genes and down-regulated a set of metastasis promoting genes. Of these genes, tissue inhibitor of metalloproteinase 2 (TIMP2) and TIMP4, which are metastatic suppressor genes, increased 8.7- and 2.5-fold in scriptaid-treated cells, respectively. SERPINE1 (PAI1), which suppresses tumor invasion through protease inhibition in stroma, was upregulated by 2.1-fold. Metastasis suppressor genes such as MTSS1, CAV1, CTSD and thrombospondin 1 were also upregulated by 2.0 -fold or higher by scriptaid. The levels of the metastasis promoting genes Neuregulin (NRG1) and Neuropolin 2 (NRP2), were decreased in scriptaid-treated cells. Transcription of NRG1 and NRP2 decreased 2.4- and 2.2-fold, respectively, in response to scriptaid. Integrin $\beta 1$, a subunit of $\alpha 5 \beta 1$, was downregulated by 2.1 -fold in response to scriptaid.

\section{Discussion}

Histone deacetylase inhibitors (HDACIs) are a new class of chemotherapeutic agents and have shown anticancer activity against diverse cancer types. Some of the HDACIs are in phase I and II clinical trials in patients with hematological and solid malignancies. In this study, the effects of scriptaid on cell growth, cell cycle, apoptosis and epigenetic alteration were investigated in RKO colorectal cancer cells. The scriptaid alone did not have an effect on the demethylation and reexpression of a hypermethylated p16 in RKO cells. However, scriptaid showed a synergistic effect with 5 -aza-dC on reexpression of the p16 in a dose-dependent manner in RKO cells, suggesting that full re-expression of aberrantly silenced p16 requires the complete reversal of modified histones in a euchromatic state. Recently, McGarvey et al (15) studied the re-expression of a hypermethylated and aberrantly silenced hMLH1 gene and histone modification in RKO cells. They found that demethylation of the $h M L H 1$ gene can induce gene expression in response to 5-aza-dC treatment, though some of repressive chromatin marks associated with heterochromatin were not changed in response to 5 -aza-dC. These observations 

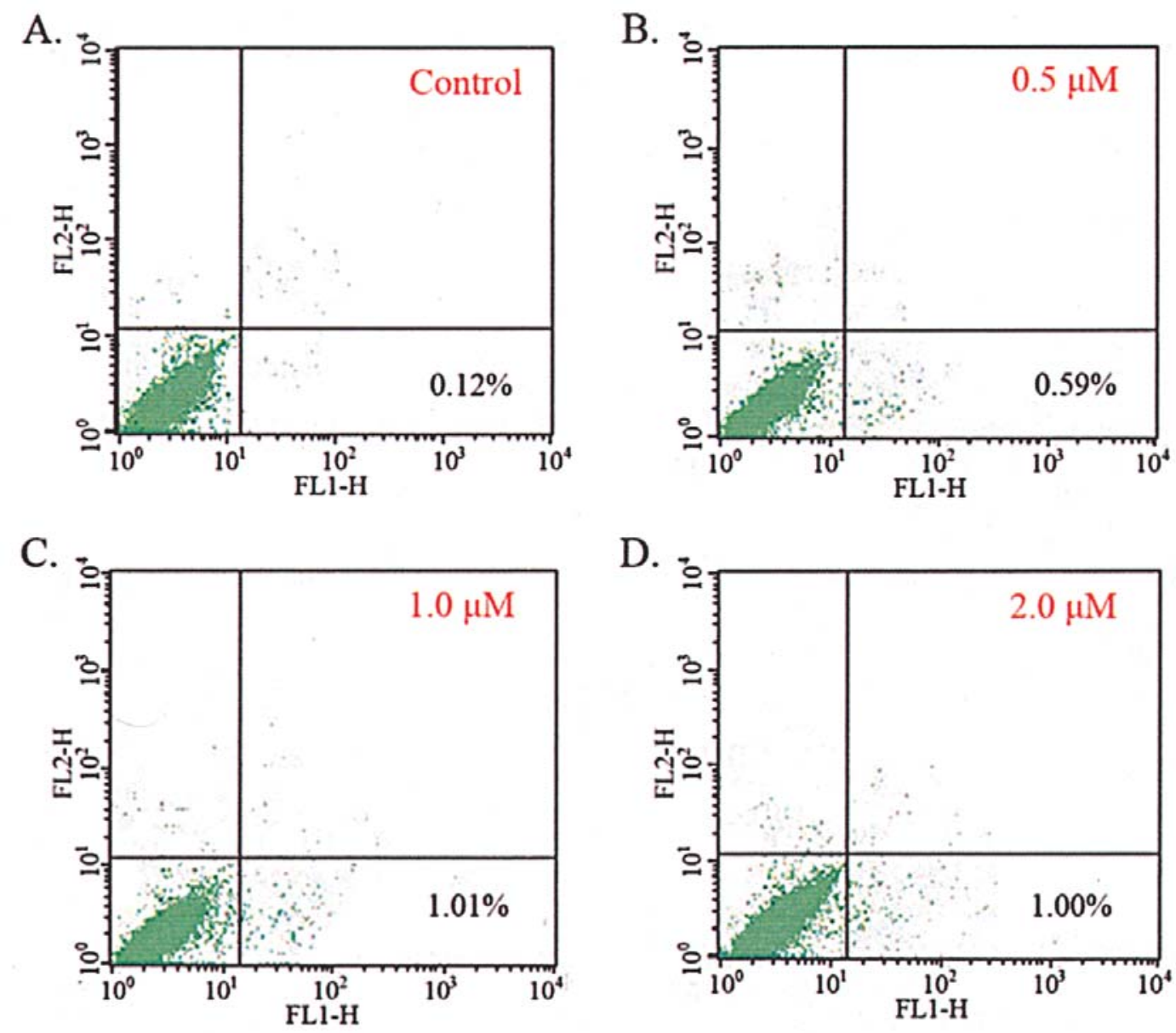

Figure 5. Apoptosis analysis of RKO cells treated with scriptaid. RKO cells were cultured with scriptaid ( $0,0.5,1.0$ and $2.0 \mu \mathrm{M})$ for 48 h. Apoptosis was detected by measuring Annexin-V protein in the cell membrane. Numbers shown indicate the percent of early apoptotic cells [Annexin-V $(+) /$ propidium iodine negative (PI-)].

suggest that 5 -aza-dC is not enough for complete reversal to the full euchromatic state seen at a normally transcribed gene at $p 16$ promoter in $\mathrm{RKO}$ cells and that scriptaid helps the complete reversal to the euchromatic state, possibly by causing histone modification.

The acetylation and methylation of histone tails in chromatin play an important role in the regulation of gene expression. The effect of scriptaid on histone modification in this study was comparable to TSA. Fahrner et al (2) reported that TSA alone leads to a slight increase in acetylated $\mathrm{H} 3$ and essentially no change in dimethyl-H3-K4 and dimethyl-H3-K9 at the hMLH1 promoter in RKO cells. Scriptaid alone led to increases in acetyl-H3-K9 and dimethyl-H3-K4 and a decrease in dimethyl-H3-K9, which is important for euchromatic gene repression at the $p 16$ promoter. The fact that scriptaid can modifiy histones regardless of the failure of demethylaton and re-expression of the hypermethylated p16 gene suggests a more dominant role of DNA methylation over histone deacetylase activity for the maintenance of gene silencing in association with hypermethylation of $\mathrm{CpG}$ island in mammals (16).

The use of 5-aza-dC alone increased the levels of acetylH3-K9 and dimethyl-H3-K4 at p16 promoter but not of dimethyl-H3-K9, a transcriptional repressive methylation mark. This observation supports the finding that histones retaining the mixture of activating and repressive chromatin marks remain in spite of the transcriptional reactivation of silenced hMLH1 in RKO cells treated with 5-aza-dC (15). Additionally, 5-aza-dC with scriptaid synergistically increased the levels of acetyl-H3-K9 and dimethyl-H3-K4 at the p16 promoter but not the level of dimethyl-H3-K9. The failure in reversal of repressive transcriptional chromatin modifications following 5-aza-dC treatment could be due to the short time course of the experiments and low dose of 5-aza-dC. Therefore, we increased the concentration and incubation time of 5-aza-dC and found an increased reversal of dimethyl-H3-K9 (data not shown), indicating that histone modification in response to 5 -aza-dC was dependent on the dosage of the compound. These results also favor the idea that dimethyl-H3-K9 may be a critical element for the complete reversal of transcription of silenced pl6 gene in RKO cells and be critical for CpG island hypermethylation and stable maintenance of heterochromatin.

Scriptaid inhibits growth of breast cancer cells and suppresses cell growth and cell cycle in endometrial and ovarian cancer cells $(11,12)$. Cell cycle analysis in RKO cells showed that scriptaid at 0.5 and $1.0 \mu \mathrm{M}$ induced $\mathrm{G} 1$ arrest and at $2.0 \mu \mathrm{M}$ increased $\mathrm{G} 1$ and $\mathrm{G} 2 / \mathrm{M}$ proportions of the cell cycle, consistent with the previous observation that SAHA at 
Table II. Gene expression profiling.

A, Genes upregulated (>2.0-fold changes) by scriptaid treatment in RKO cells

\begin{tabular}{|c|c|c|c|c|}
\hline Acc. No. ${ }^{a}$ & Gene name & Gene symbol & Chr. location & Fold change \\
\hline \multicolumn{5}{|l|}{ Cell cycle } \\
\hline NM_144665.2 & Sestrin 3 & SESN3 & $11 \mathrm{q} 21$ & 9.3 \\
\hline NM_002923.1 & Regulator of G-protein signalling 2, $24 \mathrm{kDa}$ & RGS2 & $1 \mathrm{q} 31$ & 6.1 \\
\hline NM_001992.2 & Coagulation factor II (thrombin) receptor & F2R & $5 q 13$ & 5.2 \\
\hline NM_015714.2 & G0/G1 switch 2 & GOS2 & $1 \mathrm{q} 32.2-\mathrm{q} 41$ & 3.1 \\
\hline NM_079421.2 & Cyclin-dependent kinase inhibitor 2D & CDKN2D & $19 \mathrm{p} 13$ & 3.0 \\
\hline NM_004344.1 & Centrin, EF-hand protein 2 & CENT2 & $\mathrm{Xq} 28$ & 2.9 \\
\hline NM_002048.1 & Growth arrest-specific 1 & GAS1 & $9 q 21.3-q 22$ & 2.7 \\
\hline NM_133509.2 & RAD51-like 1 (S. cerevisiae) & RAD51L1 & $14 q 23-q 24.2$ & 2.4 \\
\hline NM_001946.2 & Dual specificity phosphatase 6 & DUSP6 & $12 \mathrm{q} 22-\mathrm{q} 23$ & 2.2 \\
\hline NM_003236.1 & Transforming growth factor, $\alpha$ & TGFA & $2 \mathrm{p} 13$ & 2.3 \\
\hline NM_145733.1 & Septin 3 & Septin 3 & $22 q 13.2$ & 2.1 \\
\hline NM_005197.2 & Checkpoint suppressor 1 & CHES1 & $14 \mathrm{q} 31.3$ & 2.1 \\
\hline \multicolumn{5}{|l|}{ Metastasis } \\
\hline NM_003255.3 & Tissue inhibitor of metalloproteinase 2 & TIMP2 & $17 \mathrm{q} 25$ & 8.7 \\
\hline NM_006981.2 & Nuclear receptor subfamily 4 , group A, member 3 & NR4A3 & $9 q 22$ & 6.3 \\
\hline NM_014751.2 & Metastasis suppressor 1 & MTSS1 & $8 \mathrm{p} 22$ & 3.4 \\
\hline NM_003246.2 & Thrombospondin 1 & THBS1 & $15 q 15$ & 2.8 \\
\hline NM_003256.2 & TIMP metallopeptidase inhibitor 4 & TIMP4 & $3 \mathrm{p} 25$ & 2.5 \\
\hline NM_001753.3 & Caveolin 1 & CAV1 & 7q31.1 & 2.4 \\
\hline NM_000210.1 & Integrin, $\alpha 6$ & ITGA6 & $2 q 31.1$ & 2.3 \\
\hline NM_000099.2 & Cystatin C (amyloid angiopathy and cerebral hemorrhage) & CST3 & 20p11.21 & 2.3 \\
\hline NM_002231.2 & Kangai $1(\mathrm{CD} 82)$ & KAI1 & $11 \mathrm{p} 11.2$ & 2.2 \\
\hline NM_000602.1 & Serpine peptidase inhibitor, clade E, member 1 & SERPINE1 & $7 q 21.3-q 22$ & 2.1 \\
\hline NM_001909.3 & Cathepsin D & CTSD & $11 \mathrm{p} 15.5$ & 2.1 \\
\hline
\end{tabular}

B, Genes downregulated ( $>2.0$-fold changes) by scriptaid treatment in RKO cells

\begin{tabular}{|c|c|c|c|c|}
\hline Acc. No. ${ }^{a}$ & Gene name & Gene symbol & Chr. location & Fold char \\
\hline \multicolumn{5}{|l|}{ Cell cycle } \\
\hline BF131656 & Nucleophosmin (nucleolar phosphoprotein B23) & NPM1 & $5 q 35$ & -9.7 \\
\hline NM_199254.1 & $\begin{array}{l}\text { Transmembrane phosphoinositide 3-phosphatase and } \\
\text { tensin homolog } 2\end{array}$ & TPTE2 & $13 q 12.11$ & -9.7 \\
\hline NM_005372.1 & V-mos moloney murine sarcoma viral oncogene homolog & MOS & $8 \mathrm{q} 11$ & -3.8 \\
\hline BM907775 & $\begin{array}{l}\text { Chromosome condensation-related SMC-associated } \\
\text { protein } 1\end{array}$ & CNAP1 & $12 \mathrm{p} 13.3$ & -3.6 \\
\hline NM_004523.2 & KIF11 kinesin family member 11 & KIF11 & $10 \mathrm{q} 24.1$ & -2.9 \\
\hline NM_022346.3 & Chromosome condensation protein $\mathrm{G}$ & HCAP-G & $4 \mathrm{p} 15.33$ & -2.9 \\
\hline NM_006603.3 & Stromal antigen 2 & STAG2 & $\mathrm{Xq} 25$ & -2.8 \\
\hline NM_004701.2 & Cyclin B2 & $\mathrm{CCNB} 2$ & $15 \mathrm{q} 22.2$ & -2.6 \\
\hline NM_004702.2 & Cyclin E2 & CCNE2 & $8 \mathrm{q} 22.1$ & -2.3 \\
\hline NM_001254.3 & CDC6 cell division cycle 6 homolog & CDC6 & $17 \mathrm{q} 21.3$ & -2.2 \\
\hline NM_006101.1 & Kinetochore associated 2 & KNTC2 & $18 \mathrm{p} 11.32$ & -2.2 \\
\hline NM_004642.2 & CDK2-associated protein 1 & CDK2AP1 & $12 \mathrm{q} 24.31$ & -2.2 \\
\hline AK057663 & Anaphase promoting complex subunit 1 & ANAPC1 & $2 q 12.1$ & -2.2 \\
\hline NM_001949.2 & E2F transcription factor 3 & E2F3 & $6 \mathrm{p} 22$ & -2.1 \\
\hline NM_018685.2 & Anilin, actin binding protein & ANLN & $7 \mathrm{p} 15-\mathrm{p} 14$ & -2.1 \\
\hline
\end{tabular}


Table IIB. Continued.

\begin{tabular}{lllll}
\hline Acc. No. ${ }^{\mathrm{a}}$ & \multicolumn{1}{c}{ Gene name } & Gene symbol & Chr. location & Fold change \\
\hline Cell cycle & & & \\
NM_001258.1 & Cyclin-dependent kinase 3 & CDK3 & 17q22-qter & -2.1 \\
NM_001826.1 & CDC28 protein kinase regulatory subunit 1B & CKS1B & 1q21.2 & -2.1 \\
NM_019084.2 & Cyclin J & CCNJ & 10pter-q26.12 & -2.1 \\
NM_001418.1 & Eukaryotic translation initiation factor 4 $\gamma, 2$ & EIF4G2 & 11 15 & -2.1 \\
Metastasis & & & & -9.5 \\
NM_001334.2 & Cathepsin O & CTSO & 4q31-q32 & -9.5 \\
NM_002427.2 & Matrix metallopeptidase 13 (collagenase 3) & MMP13 & $11 \mathrm{q} 22.3$ & -2.4 \\
AF176921 & Neuregulin 1 (NRG1) & NRG1 & 8p21-p12 & -2.3 \\
BX648430 & Catenin (cadherin-associated protein), B1, 88 kDa & CTNNB1 & 3p21 & -2.3 \\
NM_003177.3 & Spleen tyrosine kinase & SYK & 9q22 & -2.2 \\
NM_182925.1 & Fms-related tyrosine kinase 4 & FLT4 & 5q34-q35 & -2.2 \\
NM_018534.3 & Neuropolin 2 (NRP2) & NRP2 & 2q33.3 & -2.1 \\
NM_003379.3 & Villin 2 (ezrin) & VIL2 & 6q25.2-q28 & -2.1 \\
NM_002211.2 & Integrin B1 & ITGB1 & 10q11.2 \\
\hline
\end{tabular}

${ }^{\mathrm{a} A c c}$ no. GenBank accession number. Chr, chromosome.

concentration of 2.5 and $5 \mu \mathrm{M}$ arrests $\mathrm{T} 24$ cells predominately in G1, whereas SAHA at higher concentrations arrests the cells in both G1 and G2 (17). These observations suggest that cell cycle arrest depends on the concentration of HDACIs. HDACI-induced cell cycle arrest and growth inhibition are largely associated with transcriptional inhibition of cyclin A and cyclin $\mathrm{D}$, or with transcriptional activation of $\mathrm{p} 21^{\mathrm{WAF} 1 / \mathrm{CIP} 1}$, p $27^{\mathrm{KIP} 1}$ and GADD $45 \alpha$ (reviewed in ref. 18). However, the expression levels of those proteins in this study did not change significantly after scriptaid treatment. Instead, expression levels of p14 and GADD45G increased and those of cyclin B2 and cyclin E2 decreased after scriptaid treatment, suggesting that mechanisms underlying cell cycle arrest and growth inhibition by scriptaid may be different from other HDACIs. The difference in the selective alteration of transcription of a gene by HDACIs may result from the different HDAC substrates and the different composition and configuration of proteins in the transcription factor complex including the HDACIs.

HDACIs have been recently shown to be important factors in cell migration and invasion for both normal and malignant cells (8). In this study, scriptaid up-regulated metastasis suppressor genes and down-regulated metastasis promoting genes. KAL1, a metastasis suppressor gene on human chromosome $11 \mathrm{p} 11.2$, is able to inhibit the progression of tumor metastasis without affecting primary tumorigenicity (19) and was presently induced by 2.2 -fold in response to scriptaid. Neurogulin 1 (NRG1) is expressed by vascular endothelial cells and regulates angiogenesis by mechanisms involving paracrine up-regulation of VEGF-A (20). Neuropolin 2 (NPR2) contributes to metastasis by interacting with the vascular endothelial growth factor (VEGF). The expression of NRG1 and NPR2 were downregulated by 2.0 -fold higher by scriptaid. Integrin $\alpha 5 \beta 1$ participates in the activation of both FLT4 (FLT4 fms-related tyrosine kinase 4: VEGFR3) and its downstream PI3 kinase/Akt signaling pathway, which is essential for lymphatic endothelial cell proliferation (21). Integrin $\alpha 5 \beta 1$ also suppresses tumor lymphangiogenesis and lymph node metastasis by blocking FLT4. Based on these observations, it is likely that scriptaid may suppress metastasis by regulating metastasis-related genes in favor of inhibiting metastasis.

Recent studies using cDNA microarrays showed that approximately $7-10 \%$ of genes were detected with altered transcription in cell lines using 2-fold change as a cut-off value. In this study, altered transcription of 2.0 -fold or higher was detected in $278(0.8 \%)$ of the 30968 genes analyzed. These fewer changes in gene transcription in response to scriptaid may result from short time of culture and low concentration of scriptaid (22-25). HDACIs can selectively alter gene transcription partly by inducing chromatin remodeling and by changing the structure of proteins in transcription factor complexes and by regulating the target genes in an affected signal transduction pathway (26). Further studies are needed to understand the mechanism(s) underlying transcriptional changes by scriptaid. In conclusion, our data suggest that scriptaid may induce growth inhibition, cell cycle arrest and histone modifications in colorectal cancer cells.

\section{Acknowledgements}

We thank Ms. Sujeong Ahn for FACS analysis mentioned in the study. This study was supported by grants from the Seoul R\&BD program (\#10582), the SRC/ERC program of MOST/KOSEF (R11-2005-017) and the Research Program 
of dual regulation mechanisms of aging and cancer from KOSEF (M1075604000107N560400110).

\section{References}

1. Esteller M, Corn PG, Baylin SB and Herman JG: A gene hypermethylation profile of human cancer. Cancer Res 61: 3225-3229, 2001

2. Fahrner JA, Eguchi S, Herman JG and Baylin SB: Dependence of histone modifications and gene expression on DNA hypermethylation in cancer. Cancer Res 62: 7213-7218, 2002.

3. Nguyen CT, Weisenberger DJ, Velicescu M, Gonzales FA, Lin JC, Liang G and Jones PA: Histone H3-lysine 9 methylation is associated with aberrant gene silencing in cancer cells and is rapidly reversed by 5 -aza-2'-deoxycytidine. Cancer Res 62: 6456-6461, 2002

4. Bachman KE, Park BH, Rhee I, et al: Histone modifications and silencing prior to DNA methylation of a tumor suppressor gene. Cancer Cell 3: 89-95, 2003.

5. Nakayama J, Rice JC, Strahl BD, Allis CD and Grewal SI: Role of histone H3 lysine 9 methylation in epigenetic control of heterochromatin assembly. Science 292: 110-113, 2001.

6. Sims RJ III, Nishioka K and Reinberg D: Histone lysine methylation: a signature for chromatin function. Trends Genet 19: 629-639, 2003.

7. Kondo Y, Shen LL and Issa JP: Critical role of histone modification in tumor suppressor gene silencing in colorectal cancer. Mol Cell Biol 23: 206-215, 2003.

8. Liu T, Kuljaca S, Tee A and Marshall GM: Histone deacetylase inhibitors: multifunctional anticancer agents. Cancer Treat Rev 32: 157-165, 2006.

9. Monneret C: Histone deacetylase inhibitors. Eur J Med Chem 40: $1-13,2005$.

10. Su GH, Sohn TA, Ryu B and Kern SE: A novel histone deacetylase inhibitor identified by high-throughput transcriptional screening of a compound library. Cancer Res 60: 3137-3142, 2000.

11. Keen JC, Yan L, Mack KM, Pettit C, Smith D, Sharma D and Davidson NE: A novel histone deacetylase inhibitor, scriptaid, enhances expression of functional estrogen receptor alpha (ER) in ER negative human breast cancer cells in combination with 5-aza 2'-deoxycytidine. Breast Cancer Res Treat 81: 177-186, 2003.

12. Takai N, Ueda T, Nishida M, Nasu K and Narahara H: A novel histone deacetylase inhibitor, Scriptaid, induces growth inhibition, cell cycle arrest and apoptosis in human endometrial cancer and ovarian cancer cells. Int J Mol Med 17: 323-329, 2006.

13. Herman JG, Graff JR, Myöhänen S, Nelkin BD and Baylin SB: Methylation-specific PCR: a novel PCR assay for methylation status of CpG islands. Proc Natl Acad Sci USA 93: 9821-9826, 1996.
14. Yang YH, Dudoit S, Luu P, Lin DM, Peng V, Ngai J and Speed TP: Normalization for cDNA microarray data: a robust composite method addressing single and multiple slide systematic variation. Nucleic Acids Res 30: el5, 2002.

15. McGarvey KM, Fahrner JA, Greene E, Martens J, Jenuwein T and Baylin SB: Silenced tumor suppressor genes reactivated by DNA demethylation do not return to a fully euchromatic chromatin state. Cancer Res 66: 3541-3549, 2006.

16. Cameron EE, Bachman KE, Myöhänen S, Herman JG and Baylin SB: Synergy of demethylation and histone deacetylase inhibition in the re-expression of genes silenced in cancer. Nat Genet 21: 103-107, 1999

17. Richon VM, Sandhoff TW, Rifkind RA and Marks PA: Histone deacetylase inhibitor selectively induces p21WAF1 expression and gene-associated histone acetylation. Proc Natl Acad Sci USA 97: 10014-10019, 2000.

18. Johnstone RW and Licht JD: Histone deacetylase inhibitors in cancer therapy: is transcription the primary target? Cancer Cell 4: 13-18, 2003.

19. Dong JT, Lamb PW, Rinker-Schaeffer CW, Vukanovic J, Ichikawa T, Isaacs JT and Barrett JC: KAI1, a metastasis suppressor gene for prostate cancer on human chromosome 11p11.2. Science 268: 884-886, 1995.

20. Iivanainen E, Paatero I, Heikkinen SM, et al: Intra- and extracellular signaling by endothelial neuregulin-1. Exp Cell Res 313: 2896-2909, 2007.

21. Zhang X, Groopman JE and Wang JF: Extracellular matrix regulates endothelial functions through interaction of VEGFR-3 and integrin alpha5beta1. J Cell Physiol 202: 205-214, 2005.

22. Chambers AE, Banerjee S, Chaplin T, Dunne J, Debernardi S, Joel SP and Young BD: Histone acetylation-mediated regulation of genes in leukaemic cells. Eur J Cancer 39: 1165-1175, 2003.

23. Mitsiades CS, Mitsiades NS, McMullan CJ, et al: Transcriptional signature of histone deacetylase inhibition in multiple myeloma: biological and clinical implications. Proc Natl Acad Sci USA 101: 540-545, 2004

24. Sasakawa Y, Naoe Y, Sogo N, et al: Marker genes to predict sensitivity to FK228, a histone deacetylase inhibitor. Biochem Pharmacol 69: 603-616, 2005.

25. Glaser KB, Staver MJ, Waring JF, Stender J, Ulrich RG and Davidsen SK: Gene expression profiling of multiple histone deacetylase (HDAC) inhibitors: defining a common gene set produced by HDAC inhibition in T24 and MDA carcinoma cell lines. Mol Cancer Ther 2: 151-163, 2003.

26. Gui CY, Ngo L, Xu WS, Richon VM and Marks PA: Histone deacetylase (HDAC) inhibitor activation of p21WAF1 involves changes in promoter-associated proteins, including HDAC1. Proc Natl Acad Sci USA 101: 1241-1246, 2004. 